\title{
CORTO CIRCUITO PULMONAR, ÍNDICE ARTERIO-ALVEOLAR Y GRADIENTE ALVEOLO-ARTERIAL DE OXÍGENO PREVIA VENTILACIÓN MECÁNICA NO INVASIVA
}

Giovane Mendieta Izquierdo ${ }^{1}$, Ana Isabel García Muñoz ${ }^{2}$, Sol Angie Romero Díaz ${ }^{3}$

\section{Resumen}

Objetivo. Describir el comportamiento de gradiente alveolo-arterial oxígeno (Gra$\mathrm{d}[\mathrm{A}-\mathrm{a}] \mathrm{O} 2)$, índice arterio-alveolar de oxígeno (Ind[a-A]O2) y corto circuito pulmonar (Qs/Qt) en un grupo de pacientes previa instauración de ventilación mecánica no invasiva -VMNI- en una unidad de cuidado intensivo UCI.

Metodología. Estudio transversal retrospectivo. Calculamos el Grad[A-a]O2) mediante la ecuación: $\mathrm{PAO} 2=[[(\mathrm{pAtm}-\mathrm{pH} 2 \mathrm{O}) \mathrm{xFiO} 2]-(\mathrm{pCO} / \mathrm{RespQ})]-\mathrm{PaO} 2]$, para: RespQ $=(0,8) ;(p A t m=560 \mathrm{mmHg}$ para Bogotá, 2640 metros sobre nivel del mar $)$; $\mathrm{pH} 2 \mathrm{O}=(47 \mathrm{mmHg})$; el (Ind[a-A]O2) y (Qs/Qt) con la fórmula: $\left(\mathrm{Cc}_{\mathrm{o} 2}-\mathrm{Ca}_{\mathrm{o} 2}\right) /\left(\mathrm{Cc}_{\mathrm{o} 2}-\right.$ $\left.\mathrm{CV}_{\mathrm{o} 2}\right)$. Ámbito: Hospital terciario. Pacientes: 16 sujetos de UCI mediante muestra no probabilística consecutiva, que recibieron VMNI en un periodo de medición de 3 meses. Variables de interés principal: diagnóstico, edad, gasometría sanguínea, se calcularon las variables: (Grad [A-a]O2), (Ind[a-A]O2) y (Qs/Qt).

Resultados. El promedio de edad de la población objeto de estudio fue de 59,6; de los cuales el 56\% $(n=9)$ fueron mujeres. La VMNI se utilizó en el $14 \%(n=16)$ de los pacientes con una población total de 116 en un periodo de tres meses. Los valores gasométricos encontrados (media \pm desviación estándar) fueron: (Ind [a-A]O2)= $(0,38 \mathrm{mmHg} \pm 0,2 \mathrm{mmHg}),(\mathrm{Qs} / \mathrm{Qt})=(25 \% \pm 8.1 \%)$ y $(\mathrm{Grad}[\mathrm{A}-\mathrm{a}] \mathrm{O} 2)=(159 \mathrm{mmHg}$ $\pm 64.1 \mathrm{mmHg}), \mathrm{FiO} 2$ suministrada de acuerdo a los requerimientos de cada paciente, $\mathrm{PaCO} 2(42 \mathrm{mmhg} \pm 15 \mathrm{mmHg}), \mathrm{PaO} 2 / \mathrm{FiO} 2=(149 \pm 44.2), \mathrm{SvO} 2 \quad(\mathrm{n}=8)=(66,2 \% \pm$ $9.3 \%), \mathrm{PH}=(7,38 \pm 0.1), \mathrm{SaO} 2=(92 \% \pm 2.8 \%), y \mathrm{PaO} 2=(70 \mathrm{mmHg} \pm 15.4 \mathrm{mmHg})$.

Conclusiones. Los valores gasométricos (Qs/Qt $=>25 \pm 8.1 \%$ ), (Ind $[\mathrm{a}-\mathrm{A}] \mathrm{O} 2=<0,38$ $\pm 0.2 \mathrm{mmHg})$ y $(\mathrm{Grad}[\mathrm{A}-\mathrm{a}] \mathrm{O} 2=>159 \pm 64.1 \mathrm{mmHg})$, identificados en este grupo de pacientes, pueden ser tenidos en cuenta previa instauración de VMNI en sujetos con características similares a una altura de 2.640 metros sobre el nivel del mar.

Palabras clave: respiración artificial, análisis de los gases de la sangre, cuidados críticos.

${ }^{1}$ PhD en Salud Pública, TR. Profesor investigador Universidad Militar Nueva Granada. Bogotá Colombia. giovane.mendieta@unimilitar.edu.co ORCID iD http://orcid.org/0000-0002-5085-3242.

${ }^{2}$ Magister en Desarrollo Educativo y Social, TR. Investigador Escuela Militar de Cadetes General José María Córdova. Bogotá Colombia.

${ }^{3}$ Magister en Educación, TR. Profesor Investigador Universidad Manuela Beltrán. Bogotá Colombia. 


\section{INTRAPULMONARY SHUNT, ALVEOLAR- ARTERIAL INDEX AND ALVEOLAR -ARTERIAL OXYGEN GRADIENT PRIOR NON-INVASIVE MECHANICAL VENTILATION}

Giovane Mendieta Izquierdo, Ana Isabel García Muñoz, Sol Angie Romero Díaz

\section{Abstract}

Objective. Describe the behavior of the alveolar-arterial oxygen gradient (Grad [Aa] $\mathrm{O} 2$ ), arterial- alveolar oxygen index (Ind [aA] O2) and Intrapulmonary shunt (Qs / $\mathrm{Qt}$ ) in a patient group prior to ventilation -VMNI- in an intensive care unit -UCI-

Methodology. Retrospective cross-sectional study. We calculate the Grad[A-a] O2 by the equation: $\mathrm{PAO} 2=[[(\mathrm{pAtm}-\mathrm{pH} 2 \mathrm{O}) \mathrm{xFiO} 2]-(\mathrm{pCO} 2 /$ RespQ $)]-\mathrm{PaO} 2]$, for: Res$\mathrm{pQ}=(0,8) ;(\mathrm{PAtm}=560 \mathrm{mmHg}$ for Bogotá, which is 2640 meters above sea level $) ; \mathrm{PH}$ $2 \mathrm{O}=(47 \mathrm{mmHg})$; The (Ind [a-A] O2) and (Qs / Qt) with the formula: $\left(\mathrm{Cc}_{\mathrm{o} 2}-\mathrm{Ca}_{\mathrm{o} 2}\right)$ / $\left(\mathrm{Cc}_{\mathrm{o} 2}-\mathrm{Cv}_{\mathrm{o} 2}\right)$. Scope: Tertiary Hospital. Patients: 16 ICU subjects using a non-probabilistic consecutive sample, who received NIMV in a 3-month measurement period. Variables of main interest: Diagnosis, age, blood gas analysis, the variables were: (Grad [A-a] O2), (Ind [a-A] O2) and (Qs / Qt).

Results. The mean age of the study population was 59.6; of these, $56 \%(n=9)$ were women. NIMV was used in $14 \%(n=16)$ of patients with a total population of 116 over a three-month period. The gasometric values found were: $($ Ind $[\mathrm{aA}] \mathrm{O} 2)=$ $(0.38 \mathrm{mmHg} \pm 0.2 \mathrm{mmHg}),(\mathrm{Qs} / \mathrm{Qt})=(25 \% \pm 8.1 \%)$ and $(\mathrm{Grad}[\mathrm{Aa}] \mathrm{O} 2), \mathrm{PaO} 2$ $42 \mathrm{mmHg} \pm 15 \mathrm{mmHg}), \mathrm{PaO} 2 / \mathrm{FiO} 2=(149 \pm 44.2), \mathrm{SvO} 2(\mathrm{n}=8)=(66.2 \% \pm) 9.3 \%)$, $\mathrm{PH}=(7.38 \pm 0.1), \mathrm{SaO} 2=(92 \% \pm 2.8 \%)$ and $\mathrm{PaO} 2(70 \mathrm{mmHg} \pm 15.4 \mathrm{mmHg})$.

Conclusions. The gasometric values (Qs / $\mathrm{Qt}=25 \pm 8.1 \%$ ), (Ind $[\mathrm{aA}] \mathrm{O} 2=<0.38 \pm$ $0.2 \mathrm{mmHg}$ ) and (Grad [Aa] $\mathrm{O} 2=159 \pm 64.1 \mathrm{MmHg}$ ), identified in this group of patients, can be taken into account prior to the onset of NIMV in subjects with similar characteristics at a height of 2,640 meters above sea level. 


\section{CURTO CIRCUITO PULMONAR, ÍNDICE ARTÉRIO-ALVEOLAR E GRADIENTE ALVÉOLO-ARTERIAL DE OXIGÊNIO APÓS VENTILAÇÃO MECÂNICA NÃO-INVASIVA}

Giovane Mendieta Izquierdo, Ana Isabel García Muñoz, Sol Angie Romero Díaz

\section{Resumo}

Objetivo. Descrever o comportamento do gradiente alvéolo-arterial de oxigênio (Grad[A-a]O2), índice artério-alveolar de oxigênio (Ind[a-A]O2) e curto-circuito pulmonar (Qs/Qt) em um grupo de pacientes após a introdução de ventilação mecânica não-invasiva, VMNI, em uma unidade de cuidados intensivos, UCI.

Metodologia. Estudo transversal retrospectivo. Nós calculamos o Grad[A-a]O2) através da equação: $\mathrm{PAO} 2=[[(\mathrm{pAtm}-\mathrm{pH} 2 \mathrm{O}) \times \mathrm{FiO} 2]-(\mathrm{pCO} 2 / \mathrm{RespQ})]-\mathrm{PaO} 2]$, para: RespQ $=(0,8) ;(p A t m=560 \mathrm{mmHg}$ para Bogotá, 2640 metros acima do nível do mar); $\mathrm{pH} 2 \mathrm{O}=(47 \mathrm{mmHg})$; o (Ind $[\mathrm{a}-\mathrm{A}] \mathrm{O} 2)$ e $(\mathrm{Qs} / \mathrm{Qt})$ com a fórmula: $\left(\mathrm{Cc}_{\mathrm{o} 2}-\mathrm{Ca}_{\mathrm{o} 2}\right) /$ $\left(\mathrm{Cc}_{\mathrm{o} 2}-\mathrm{C}_{\mathrm{o} 2}\right)$. Ámbito: Hospital terciário. Pacientes: 16 sujeitos de UCI mediante amostra não probabilística consecutiva, que receberam VMNI por um período de medição de 3 meses. Variáveis de interesse principal: diagnóstico, idade, gasometria de sangue, as variáveis (Grad [A-a]O2), (Ind[a-A]O2) e (Qs/Qt) foram calculadas.

Resultados. a idade média da população alvo foi de 59,6; $56 \%(n=9)$ eram mulheres. VMNI foi usada em 14\% $(n=16)$ dos pacientes com uma população total de 116 durante um período de três meses. Foram encontrados os seguintes valores de gasometria (média \pm desvio-padrão): (Ind [a-A]O2) $=(0,38 \mathrm{mmHg} \pm 0,2 \mathrm{mmHg}),(\mathrm{Qs} /$ $\mathrm{Qt})=(25 \% \pm 8.1 \%)$ e $(\mathrm{Grad}[\mathrm{A}-\mathrm{a}] \mathrm{O} 2)=(159 \mathrm{mmHg} \pm 64.1 \mathrm{mmHg})$, FiO2 fornecida de acordo com os requisitos de cada paciente, $\mathrm{PaCO} 2(42 \mathrm{mmhg} \pm 15 \mathrm{mmHg}), \mathrm{PaO} 2 /$ $\mathrm{FiO} 2=(149 \pm 44.2), \mathrm{SvO} 2(\mathrm{n}=8)=(66,2 \% \pm 9.3 \%), \mathrm{PH}=(7,38 \pm 0.1), \mathrm{SaO} 2=(92 \%$ $\pm 2.8 \%)$, e $\mathrm{PaO} 2=(70 \mathrm{mmHg} \pm 15.4 \mathrm{mmHg})$.

Conclusões. os valores de gasometria (Qs/Qt $=>25 \pm 8.1 \%$ ), (Ind[a-A]O2 $=<0,38$ $\pm 0.2 \mathrm{mmHg})$ e $(\mathrm{Grad}[\mathrm{A}-\mathrm{a}] \mathrm{O} 2=>159 \pm 64.1 \mathrm{mmHg})$, identificados neste grupo de pacientes podem ser tomados em consideração após implementação de VMNI em sujeitos com características semelhantes a uma altura de 2.640 metros acima do nível do mar. 


\section{Introducción}

El uso de la VMNI en el tratamiento de insuficiencia respiratoria aguda y crónica ha aumentado en los últimos años (13) demostrando disminución en la mortalidad y reducción de la estancia media en UCI y de la necesidad de intubación $(4,5)$. Otros beneficios han sido: mejoría más rápida del $\mathrm{PH}, \mathrm{PCO} 2, \mathrm{PaO} 2$, disnea y frecuencia respiratoria, así como la disminución de complicaciones y reducción de costos hospitalarios (6-8).

La VMNI es un modo usado con mayor frecuencia en exacerbaciones de EPOC que cursan con acidosis respiratoria (8-13), en edema agudo de pulmón (14-16) y en síndrome de inmunodeficiencia adquirida $(4,17,18)$. Sin embargo, se ha reportado que hasta el $20 \%$ de los hospitales europeos no emplean nunca la VMNI, mientras que en los pacientes con ventilación mecánica únicamente en el $16 \%$ se utiliza la modalidad no invasiva. En Estados Unidos la VMNI se utiliza en el 20\% de los pacientes que requieren ventilación mecánica (19).

Estudios previos, así como los consensos Argentino, Británico y Americo-Eruropeo de VMNI, reconocen su uso en sujetos con parámetros gasométricos: $\mathrm{PaCo} 2>45 \mathrm{mmhg},<300, \mathrm{PH}$ : $>7.25$ y/o $<7,35$ (20-23). Se reconoce un estudio previo en Colombia que describe la frecuencia de fracaso de la VMNI en pacientes con falla respiratoria hipercápnica secundaria a EPOC previo análisis de las variables de gasometría sanguínea: $\mathrm{PH}, \mathrm{PaCO} 2, \mathrm{HCO} 3, \mathrm{PaO} 2$, $\mathrm{SaO} 2, \mathrm{SvO} 2, \mathrm{PaFiO} 2, \mathrm{Qs} / \mathrm{Qt}$, (Gra-
$\mathrm{d}[\mathrm{A}-\mathrm{a}] \mathrm{O} 2)$ e (Ind[a-A]O2), en donde se identificó, entre otros aspectos, una $\mathrm{PaCO} 2$ mayor a $55 \mathrm{~mm} \mathrm{Hg}$ como factor de riesgo para fracaso para VMNI (24). El objetivo de este artículo es describir el comportamiento de (Grad[A-a]O2), (Ind[a-A]O2) y (Qs/Qt) en un grupo de pacientes previa instauración de VMNI en una UCI.

\section{Pacientes y métodos}

Se realizó un estudio transversal retrospectivo con una población (N) 116 y una muestra de casos consecutivos (n) 16 sujetos en una UCI. Se emplearon registros de información secundaria; para el presente estudio se usó la base de datos de "Registros de uso de VMNI" de la UCI, de la cual se extrajeron las variables de rutina registradas al momento de instaurar la VMNI: datos demográficos, edad, sexo, gasometría arterial y venosa previa instauración de la VMNI ( $\mathrm{PH}, \mathrm{PaCO} 2, \mathrm{HCO}$, $\mathrm{PaO} 2, \mathrm{SaO} 2, \mathrm{SvO} 2,(\mathrm{PaFiO} 2)$, (Qs/ $\mathrm{Qt}),(\mathrm{Grad}[\mathrm{A}-\mathrm{a}] \mathrm{O} 2),(\operatorname{Ind}[\mathrm{a}-\mathrm{A}] \mathrm{O} 2)$, así como la determinación de incidencia acumulada, mortalidad y necesidad de intubación.

Cálculos de variables primarias: el (Grad[A-a]O2) mediante la ecuación $\mathrm{PAO} 2=[[(\mathrm{pAtm}-\mathrm{pH} 2 \mathrm{O}) \times \mathrm{FiO} 2]-(\mathrm{p}-$ CO2/RespQ)]-PaO2], para RespQ = $(0,8), \mathrm{pAtm}=(560 \mathrm{mmHg}$ para Bogotá $)$ y $\mathrm{pH} 2 \mathrm{O}=(47 \mathrm{mmHg})$ menos la $\mathrm{PaO} 2$. Se consideró que el paciente estaba bien clasificado cuando su ( $\operatorname{Grad}[\mathrm{A}-\mathrm{a}]$ $\mathrm{O} 2)$ era superior al teórico, $\mathrm{AaPO} 2=$ $2,5+(0,21 \times$ edad en años) (Fórmula de Mellemgaard) (25). Para la fracción de corto circuito pulmonar Qs/Qt, 
se empleó la fórmula (Cco2- $\mathrm{Cao} 2) /$ (Cco2- Cvo2). La FiO2 suministrada al momento de la toma de las muestras se daba de acuerdo a las necesidades de cada paciente.

En el periodo comprendido del 1 de octubre al 31 de diciembre de 2009 se evaluó el "registro de uso de VMNI" de los pacientes que recibieron VMNI en la UCI de un Hospital público de tercer nivel de atención en la ciudad de Bogotá,Colombia. El procedimiento de análisis y toma de gasometría arterial se realizó de manera rutinaria, para orientar la intervención terapéutica respiratoria por parte del Terapeuta Respiratorio (TR), la toma de la gasometría venosa fue realizada por el profesional de enfermería previa instauración de la VMNI. La información fue registrada en la base datos registro de uso de VMNI en Excel por el TR que tomaba y analizaba la prueba.

El análisis de la gasometría sanguínea se realizó en la maquina marca Cobas 221 y las pruebas fueron tomadas con jeringas de gases sanguíneos de $1 \mathrm{cc}$ con heparina de litio (601) "Vital Signs" de acuerdo al protocolo establecido. Análisis de datos: se realizó de manera retrospectiva mediante el paquete estadístico IBM SPSS versión 22 por medio de la funciones de medida de tendencia central e IC. Las variables cualitativas se expresan mediante distribución de frecuencia absoluta y proporción. Las variables cuantitativas se describieron mediante la medida de tendencia central, media o mediana, y desviación estándar de la media. Consideraciones éticas: se contempló la confidencialidad de la información, así como el anonimato, elemento fundamental de ética, no se reconocen consecuencias negativas resultantes de la publicación del estudio, este estudio se enmarca en los lineamientos del Código de Helsinki (26) y la Resolución 008430/19934.

\section{Resultados}

Durante tres meses 16 pacientes, de un total de 116, recibieron soporte ventilatorio mecánico no invasivo. El promedio de edad de la población objeto de estudio fue de 59,6\%. Un total de 9 sujetos eran mujeres (56\%). La relación de la VMNI respecto a la Ventilación Mecánica Invasiva (VMI), refleja una incidencia acumulada de 13,7\%. Es así como 16 de los 116 pacientes ingresados en los tres meses recibieron VMNI (Tabla 1). En el periodo del estudio se identificaron (n) 16 pruebas de gasometría sanguínea arterial y $8(n)$ reportes de gasometría venosa registrando tan solo el valor de la $\mathrm{SvO} 2$.

\footnotetext{
${ }^{4}$ Resolución 008430/1993, de 4 de octubre por la cual se establecen las normas científicas, técnicas y administrativas para la investigación en salud. Ministerio de Salud. Disponible: http://www. minproteccionsocial.gov.co/vbecontent/library/documents/DocNewsNo267711.pdf
} 
INVESTIGACIONES ANDINA No. 33 Vol. 18

Tabla 1. Incidencia acumulada para el uso de VMNI

\begin{tabular}{|c|c|c|c|c|}
\hline & $\begin{array}{c}\text { Mes } 1 \\
\mathrm{n}\end{array}$ & $\begin{array}{c}\text { Mes } 2 \\
\mathrm{~N}\end{array}$ & $\begin{array}{c}\text { Mes } 3 \\
\mathrm{n}\end{array}$ & $\begin{array}{c}\text { Total } \\
\mathrm{N}\end{array}$ \\
\hline $\begin{array}{l}\text { Pacientes con Ventilación } \\
\text { Mecánica Invasiva VMI }\end{array}$ & 40 & 37 & 23 & 100 \\
\hline $\begin{array}{l}\text { Pacientes en Ventilación } \\
\text { mecánica no invasiva VMNI }\end{array}$ & 7 & 3 & 6 & 16 \\
\hline Total & 47 & 40 & 29 & 116 \\
\hline Incidencia acumulada & & & & $13,7 \%$ \\
\hline
\end{tabular}

Durante los 3 meses de duración del estudio, el reporte gasométrico, previo al uso de la VMNI, está indicado a los pacientes que presentaron tendencia a la hipo-ventilación alveolar PaCO2 (42 \pm 15 mmHg) y con índices de oxigenación bajos. Además de sujetos con disfunción pulmonar leve-moderada $(\mathrm{PaFiO} 2=149 \pm 44.2)$, alteración en el intercambio gaseoso severo, reflejado con (Ind [a-A] O2 = 0,38 $\pm 0.2 \mathrm{mmHg}$ ), fracción de corto circuito intrapulmonar (Qs/Qt $=25 \pm 8.1 \%) y$ (Grad [A-a] O2 = $159 \pm 64.1 \mathrm{mmHg}) . \mathrm{El}$ reporte de gasometría venosa reflejado en la $\mathrm{SvO} 2(\mathrm{n}=8)$ muestra hipoperfusión tisular $=66,2 \pm 9.3 \%$. No se evidenció alteración relevante del $\mathrm{PH}=7.38 \pm 0.11$. Ninguno de los pacientes presentó disminución de la $\mathrm{SaO} 2=92 \pm 2.8 \%$, ni de la $\mathrm{PaO} 2=70 \pm 15.4 \mathrm{mmHg}$ (Tabla 2).

Tabla 1. Incidencia acumulada para el uso de VMNI

\begin{tabular}{l|c|c|c}
\hline \multicolumn{1}{c|}{ Variable } & Frecuencia & Media & Dev Std \pm \\
\hline Gasometría sanguínea & 16 & & \\
$\mathrm{PH}$ & 16 & 7,38 & 0,11 \\
$\mathrm{PaCo} 2$ & 16 & $42 \mathrm{mmHg}$ & $15 \mathrm{mmHg}$ \\
$\mathrm{HCO} 3$ & 16 & 22,5 & 5,05 \\
$\mathrm{PaO} 2$ & 16 & $70 \mathrm{mmHg}$ & $15,4 \mathrm{mmHg}$ \\
$\mathrm{SaO} 2$ & 16 & $92 \%$ & $2,8 \%$ \\
$\mathrm{SvO2}$ & 8 & $66,2 \%$ & $9,3 \%$ \\
$\mathrm{PaFiO} 2^{*}$ & 16 & 149 & 44,2 \\
$\mathrm{Qs} / \mathrm{Qt}$ & 8 & $25 \%$ & $8,1 \%$ \\
(Grad[A-a]O2) & 16 & $159 \mathrm{mmHg}$ & $64,1 \mathrm{mmHg}$ \\
(Ind[a-A]O2) & 16 & $0,38 \mathrm{mmHg}$ & $0,2 \mathrm{mmHg}$ \\
Edad & 16 & 59,6 & 11,31 \\
\hline
\end{tabular}

$\dagger \pm$ Desviación estándar.

${ }^{\star} \mathrm{FiO} 2$ : variable de acuerdo a necesidades del paciente. 
Respecto a la necesidad de intubación IOT posterior al manejo con VMNI, el 50\% de los sujetos $(\mathrm{n}=8)$ requirió VMI por deterioro de su condición clínica, un 19\% fue reportado como fallecido $(n=3)$. En este caso, las enfermedades de los sujetos fueron: EPOC 44\% ( $\mathrm{n}=7)$, Neumonía Adquirida en la Comunidad (NAC) $19 \%(n=3)$, IAM 12,5\% ( $\mathrm{n}=2)$, Trauma Torácico, CA gástrico, Pancreatitis y TEP (Tabla 3).

Tabla 3. Patologías de pacientes con VMNI. Incidencia de IOT y mortalidad.

\begin{tabular}{l|c|c}
\hline \multicolumn{1}{c|}{ Diagnóstico } & Frecuencia & $\%$ \\
\hline EPOC & 7 & 43.7 \\
NAC & 3 & 18.7 \\
IAM & 2 & 12.5 \\
Trauma torácico & 1 & 6.2 \\
CA gástrico & 1 & 6.2 \\
Pancreatitis & 1 & 6.2 \\
TEP & 1 & 6.2 \\
Total pacientes & $\mathbf{1 6}$ & $\mathbf{9 9 . 7}$ \\
Mortalidad & $\mathbf{3}$ & $\mathbf{1 9}$ \\
Incidencia de IOT & $\mathbf{7}$ & $\mathbf{4 3 , 7}$ \\
\hline
\end{tabular}

\section{Discusión}

En este estudio se demostró que la variación de los criterios gasométricos (Qs/ $\mathrm{Qt})$, (Ind[a-A]O2) y (Grad[A-a]O2) reflejan alteración grave del intercambio gaseoso y pueden ser indicación para instaurar VMNI. La aplicación de este modo ventilatorio puede considerarse como estrategia de elección en la atención de pacientes con falla respiratoria, previo análisis de criterios gasométricos y clínicos (24).

La presencia de dos o más de los siguientes criterios gasométricos: $\mathrm{PaCo} 2$ $>45 \mathrm{mmhg}, \mathrm{PaFiO} 2<300, \mathrm{PH}:>7.25$ y/o $<7.35$, se han definido para indicar el uso de la VMNI (1-3) (20-23). El gradiente alveolo-arterial de oxígeno (Grad[A-a] O2) es una forma sencilla de medir la alteración entre el alveolo y el capilar, su uso está dado en el estudio de diferentes patologías como en EPOC y TEP (2729); en cirrosis no se reconoce su uso en la indicación de la VMNI. Se suele indicar que el valor del Grad(A-a)O2 no debe exceder los $20 \mathrm{mmHg}$ en situaciones de estabilidad hemodinámica y $\mathrm{FiO} 2$ de 0,21 (30). Nuestros pacientes contaban con daño pulmonar (Grad[A-a]O2 $=159 \mathrm{mmHg} \pm 64.1 \mathrm{mmHg}$ ), valor superior a lo reportado por Sánchez Casado, media $60 \mathrm{mmHg}$ en un grupo de pacientes con o sin daño pulmonar y con $\mathrm{PaFi}$ $>300$ (30). El aumento de la fracción de corto circuito pulmonar (Qs/QT) es el 
principal mecanismo que rige la hipoxemia arterial en sujetos con lesión pulmonar aguda $(31,32)$; en un estudio se menciona el valor basal de corto circuito pulmonar en Injuria pulmonar aguda $(20 \pm 10 \%)$ y en sujetos con EPOC un valor de (11 $\pm 3 \%)$, valor inferior a lo reportado en nuestro estudio $(25 \pm$ $8,1 \%)$, el estudio no menciona el uso del corto circuito pulmonar (Qs/Qt) para la indicación de VMNI (33). En nuestro estudio se incluyó el análisis del corto circuito pulmonar (Qs/Qt), (Grad[A-a] $\mathrm{O} 2)$, (Ind[a-A]O2), que previamente no han sido presentado para la instauración de la VMNI.

Nosotros creemos que se pueden incluir los criterios (Qs/Qt $=>25 \pm 8.1 \%$ ), (In$\mathrm{d}[\mathrm{a}-\mathrm{A}] \mathrm{O} 2=<0,38 \pm 0.2 \mathrm{mmHg})$ y (Gra$\mathrm{d}[\mathrm{A}-\mathrm{a}] \mathrm{O} 2=>159 \pm 64.1 \mathrm{mmHg}$ ), como criterios gasométricos de análisis, previa instauración de la VMNI en pacientes con características similares a una altura de 2.640 metros sobre el nivel del mar. Como se describe en estudios previos, el mayor uso de la VMNI está dado en sujetos con $\operatorname{EPOC}(3,6,8,13-16)$, aspecto que es similar a lo reportado por nosotros. En nuestro estudio se demuestra una vez más que el uso de la VMNI en las UCIs es bajo, lo que sugiere la necesidad de incrementar su utilización como estrategia ventilatoria.

El análisis gasométrico que se presentó en este estudio se dio en sujetos con hipo-ventilación alveolar, disfunción pulmonar leve-moderada, criterios descritos en estudios previos $(1,8,20,21)$. Estos criterios aportan evidencia del uso de la VMNI en la Insuficiencia Respiratoria Hipoxémica $(22,23,34-41)$.
La modalidad no invasiva en los pacientes que requieren ventilación mecánica es utilizada en proporciones bajas (16\% en Europa y 20\% en Estados Unidos) (19), que son comparables a la incidencia encontrada en nuestro estudio $(13,7 \%)$. La incidencia de IOT encontrada en nuestro estudio es del $43 \%$, superior a lo descrito por Lasdica quien reporta 26\% $(n=27)(37)$. Respecto a la incidencia de mortalidad, nosotros reportamos $19 \%(n=3)$, menor a lo reportado en los estudios de Lasdica y Meduri $25,9 \%(\mathrm{n}=27) \quad$ y $32 \%$ respectivamente $(\mathrm{n}=26)(37-38)$.

Como fortalezas del estudio se relacionan la sistematización de la toma de muestras de gasometría y su análisis, así como la inclusión de todos los pacientes que recibieron VMNI durante los 3 meses de duración del estudio. Sin embargo, es importante indicar como limitaciones del estudio el diseño y análisis estadístico, el número de pacientes incluidos, el tiempo de análisis, así como el trabajar con fuentes de información secundaria, situación que genera problemas de calidad, registro y clasificación de la información (42). Por otro lado, debido al corto tiempo de análisis no fue posible evaluar a largo plazo la evolución de los pacientes tratados con VMNI, así como su relación con el uso de la VM.

\section{Conclusión}

En nuestro estudio la indicación gasométrica de la VMNI se reportó en sujetos con hipo-ventilación alveolar, disfunción pulmonar leve-moderada, fracción de corto circuito pulmonar 
(Qs/Qt) aumentado, alteración del intercambio gaseoso severo (Ind[a-A] O2) y aumento de la (Grad[A-a]O2), sin alteración del $\mathrm{pH}$. Los valores gasométricos $(\mathrm{Qs} / \mathrm{Qt}=>25 \pm 8.1 \%)$, (Ind[a-A] $\mathrm{O} 2=<0,38 \pm 0.2 \mathrm{mmHg}$ ) y gradiente alveolo-arterial de oxígeno (Grad[A-a]O2 $=>159 \pm 64.1 \mathrm{mmHg}$ ), identificados en este grupo de pacientes, pueden ser teni-

\section{Referencias}

1. International Consensus Conferences in Intensive Care Medicine. Noninvasive positive pressure ventilation in acute respiratory failure. Am J Respir Crit Care Med. 2001;163:283-91.

2. Gacouin A, Jouneau S, Letheulle J, Kerjouan M, Bouju P, Fillatre $P$, et al. Trends in prevalence and prognosis in subjects with acute chronic respiratory failure treated with noninvasive and/or invasive ventilation. Respir Care [Internet]. 2015 [Citado el 27 de junio de 2016]; 60(2):210-8. Disponible en: https://www.scopus.com/inward/ record. uri?eid=2-s2.0-84935112297\& partnerlD=40\& $\mathrm{md} 5=76 \mathrm{e} 1 \mathrm{db} 507843 \mathrm{ecb} 7 \mathrm{f}$ $7 b 729 e 865 b 81483$

3. Fiorino $S$, Bacchi-Reggiani L, Detotto E, Battilana M, Borghi E, Denitto C, et al. Efficacy of non-invasive mechanical ventilation in the general ward in patients with chronic obstructive pulmonary disease admitted for hypercapnic acute respiratory failure and $\mathrm{pH}<7.35$ : A feasibility pilot study. Intern Med J [Internet]. 2015;45(5):527-37. Disponible en: https://www.scopus.com/inward/record. dos en cuenta al momento de establecer la VMNI en sujetos con características similares a una altura de 2.640 metros sobre el nivel del mar.

\section{Conflicto de Intereses}

Los autores declaran no tener ningún conflicto de intereses. uri?eid=2-s2.0-84928985157\&partne$\mathrm{rID}=40 \& \mathrm{md} 5=61 \mathrm{e} 3722 \mathrm{fced} 240$ eceed534aae66765cf

4. Hilbert G, Gruson D, Vargas F. Noninvasive ventilation inimmunosuppressed patients with pulmonary infiltrates, fever, and acute respiratory failure. $\mathrm{N}$ Engl Med J. 2001; 344: 481-7.

5. Piroddi IMG, Barlascini C, Esquinas A, Braido F, Banfi P, Nicolini A. Non-invasive mechanical ventilation in elderly patients: $A$ narrative review [Internet]. 2016 [Citado el 27 de junio de 2016]. Disponible en: https:// www.scopus.com/inward/record.uri?ei$\mathrm{d}=2$-s2.0-84971241636\&partnerID=40\&md5=8c39182e28ed9e0f145d53d6538c1395

6. Lightowler JV, Wedzicha JA, Elliott MW, Ram FS. Non-invasive positive pressure ventilation to treat respiratory failure resulting from exacerbations of chronic obstructive pulmonary disease: Cochrane systematic review and meta-analysis. BMJ. 2003;326:185.

7. Gray A, Goodacre S, Newby DE, Masson M, Sampson F, Nicholl J, for the 3CPO 
Trialists. Noninvasive Ventilation in Acute Cardiogenic Pulmonary Edema. N Engl J Med. 2008; 359:142-51.

8. Ferrer M, Torres A. Noninvasive ventilation for acute respiratory failure. Curr Opin Crit Care [Internet]. 2015 [Citado el 27 de junio de 2016].;21(1):1-6. Disponible en: https://www.scopus.com/inward/record. uri?eid=2-s2.0-84920930305\& partnerID $=40 \&$ md $5=2$ b 365 a $99 \mathrm{f} 3 \mathrm{afacd}-$ $56783 b 29$ e98b9879e

9. Plant PK, Owen JL, Elliott MW. One year period prevalence of respiratory acidosis in acute exacerbations of COPD: implications for the provision of non-invasive ventilation and oxygen administration. Thorax. 2000; 55: 550-4.

10. Brochard L, Mancebo J, Wysocki M, Lofaso F, Conti G, Rauss A, et al. Noninvasive ventilation for acute exacerbations of chronic obstructive pulmonary disease. N Engl J Med. 1995; 333:817-22.

11. Wedzicha JA. Noninvasive ventilation for exacerbations of respiratory failure in chronic obstructive pulmonary disease. Thorax. 1996;51(Suppl 2):35-9.

12. Plant PK, Owen JL, Parrott S, Elliott MW. Cost effectiveness of ward based non- invasive ventilation for acute exacerbations of chronic obstructive pulmonary disease: economic analysis of randomised controlled trial. BMJ. 2003;326:956.

13. Kant KM, Djamin RS, Belderbos HNA, Van Den Berg B. Acute respiratory failure due to COPD: Invasive mechanical ventilation or not? Ned Tijdschr Geneeskd [Internet]. 2014 [Citado el 27 de junio de 2016];(25):987-94. Disponible en: https://www.scopus.com/inward/record. uri?eid=2-s2.0-84904253798\& partner $I D=40 \& m d 5=e 3 d 73 c c 9 c c 994580 e-$ $608074 \mathrm{f7aa3ee64}$

14. Masip J, Betbese AJ, Páez J, Vecilla $F$, Cañizares R, Padro J, et al. Non-invasive pressure support ventilation versus conventional oxygen therapy in acute car- diogenic pulmonary edema: aran domisedtrial.Lancet.2000; 283: 235-41. 5.

15. Gray A, Goodacre S, Newby DE, Masson M, Sampson F, Nicholl J, for the 3CPO Trialists. Noninvasive Ventilation in Acute Cardiogenic Pulmonary Edema. N Engl J Med. 2008; 359:142-51.

16. Rialp Cervera G, Del Castillo Blanco A, Pérez Aizcorreta O, Parra Morais L. Noninvasive mechanical ventilation in chronic obstructive pulmonary disease and in acute cardiogenic pulmonary edema. Med Intensiva [Internet]. 2014 [Citado el 27 de junio de 2016]; 38(2):111-21. Disponible en: https://www.scopus.com/inward/record. uri?eid=2-s2.0-84894283582\&partnerID=40\&md5=fa61d14a7dac1624a50dd4f9f101 cf1e

17. González-Velásquez E, Serna-Secundino HI. Ventilación mecánica no invasiva para tratar insuficiencia respiratoria aguda en pacientes con $\mathrm{VIH}$ y tuberculosis. Neumología y Cirugía de Tórax. miliar, reporte de un caso y revisión de la literatura. 2008:67(4): 178-182.

18. Lefebvre A, Rabbat A. Noninvasive ventilation in immunocompromised patients. Reanimation [Internet]. 2015 [Citado el 27 de junio de 2016]; 24(5):586-98. Disponible en: https:// www.scopus.com/inward/record. uri?eid=2-s2.0-84942760103\&partne$r \mid D=40 \& m d 5=f 9 f 8 f b 5 d a 7727 b-$ b3aa11595a09efb743

19. Maheshwari V, Paioli D, Rothaar R, Hill $\mathrm{NH}$. Utilization of noninvasive ventilation in acute care hospitals. A regional survey. Chest. 2006; 129: 1226-33.

20. Díez AR, Abbona H, Ferrero G, Figueroa Casas JC, De Vega M, Lisanti R. Consenso Argentino de Ventilación no invasiva. Medicina 2005;65:437-457.

21. Davidson AC, Banham S, Elliott M, et al. BTS/ICS Guidelines for the Ventilatory Management of Acute Hypercapnic Respiratory Failure in Adults. Thorax. 
2016 [Citado el 27 de junio de 2016]; 71:ii1-ii35. Disponible en https://www. brit-thoracic.org.uk/document-library/clinical-information/acute-hypercapnic-respiratory-failure/bts-guidelines-for-ventilatory-management-of-ahrf/ Acceso 9 de Junio 2017.

22. Antonelli M, Conti G, Pelosi P, Gregoretti $C$, Pennisi MA, Costa $R$, et al. New treatment of acute hypoxemic respiratory failure: Noninvasive pressure support ventilation delivered by helmet---A pilot controlled trial. Crit Care Med. 2002; 30:602-8.

23. Zhu F, Liu Z-L, Long X, Wu X-D, Zhou J, Bai C-X, et al. Effect of noninvasive positive pressure ventilation on weaning success in patients receiving invasive mechanical ventilation: A meta-analysis. Chin Med J (Engl) [Internet]. 2013 [Citado el 27 de junio de 2016]; 126(7):1337-43. Disponible en: https://www.scopus.com/inward/record. uri?eid=2-s2.0-84877359871\&partn e r I D $=40 \&$ m d $5=54$ c 88 d 35 b 32fa66689aa789989a84282

24. Varón FA, Alí A, Aguirre A, González M, Hernández A, Páez N, Pardo JM. Predicción de fracaso en ventilación mecánica no invasiva en falla respiratoria en enfermedad pulmonar obstructiva crónica a grandes alturas. Acta Colombiana de Cuidado Intensivo. 2013;13 (1): 12-17.

25. Mellemgaard, K. The alveolar-arterial oxygen difference. Size and components in normal man. Acta Physiol Scand.1966; $67: 10$

26. Asociación Médica Mundial. Declaración de Helsinki de la asociación médica mundial. Principios éticos para las investigaciones médicas en seres humanos. [Internet]. 1964 [Citado el 27 de junio de 2016] Disponible en: file:///C:/Users/giovane $\% 20$ mendieta/Downloads/51DocyNormas_DeclaracionHelsinki.pdf

27. Conti V, Paone G, Mollica C, Sebastiani A, Mannocci A et al. Predictors of outcome for patients with severe respiratory failure requiring noninvasive mechanical ventilation. European Review for Medical and Pharmacological Sciences 2015; 19: 3855-3860.

28. Stein PD, Goldhaber SZ, Henry JW. Alveolar-arterial oxygen gradient in the assessment of acute pulmonary embolism. Chest. 1995; 107:139-143.

29. Cervera R, del Castillo Blanco, Pérez Aizcorreta O, Parra Morais L. Puesta al día en medicina intensiva: ventilación mecánica en diferentes entidades. Ventilación mecánica no invasiva en la enfermedad pulmonar obstructiva crónica y en el edema agudo de pulmón cardiogénico. Med Intensiva. 2014; 38(2):111-121.

30. Sánchez Casado et al. Relación entre el gradiente alveolo-arterial de oxígeno y la $\mathrm{PaO} 2 / \mathrm{FiO} 2$ introduciendo la PEEP en el modelo. Med Intensiva. 2012; 36(5):329334.

31. Lemaire, F., D. Matamis, N. Lampron, B. Teisseire, and A. Harf. Intrapulmonary shunt is not increased by $100 \%$ oxygen ventilation in acute respiratory failure. Bull. Eur. Physiopathol. Respir. 1985. 21:251-256.

32. Manzo-Palacios E, Mayo-Mendoza RF, De la Cruz-López J. La corrección del índice de oxigenación en los pacientes críticos al nivel de la ciudad de México. Revista de la Asociación Mexicana de Medicina Crítica y Terapia Intensiva. 2008:XXXII(1):26-36.

33. Santos C, Ferrer M, Roca J, Torres A, Hernandez C, Rodriguez-Roisin R. Pulmonary Gas Exchange Response to Oxygen Breathing in Acute Lung Injury: Hyperoxia, Gas Exchange, and Acute Respiratory Failure. Am J Respir Crit Care Med 2000;161:(1):26-31

34. Dantzker, D. R., C. J. Brook, P. Dehart, J. P. Lynch, and J. G. Weg. Ventilationperfusion distributions in the adult respiratory distress syndrome. Am. Rev. Respir. 
35. Ferrer $M$, Esquinas $A$, León $M$, González G, Alarcón A, Torres A. Noninvasive ventilation insevere hypoxemic respiratory failure: arandomized clinical trial. Am J RespirCritCareMed. 2003;168:1438-44.

36. Principi T, Pantanetti S, Catani F, Elisei D, Gabbanelli V, Pelaia A, et al. Non-invasive continuous positive airway pressure delivered by helmet in hematological malignacy patients with hypoxemic acute respiratory failure. Intensive Care Med. 2004;30:147---50.

37. Lasdica S, Fainsteis D, Casas P, Frizza I, Ontivero M, Giussani J P, Urizar R. Ventilación mecánica no invasiva en la lesión pulmonar aguda hipoxémica. Med Intensiva. 2001;25:(8)303-309.

38. Meduri U, Turner R, Abou-Shala N. Noninvasive positive pressure ventilation via face mask. First-Line intervention in patients with acute hypercapnic and hypoxemic respiratory failure. Chest 1996; 109: 179-193.37

39. Rocker G, Mackenzie M, Williams B, Logan M. Noninvasive positive pressure ventilation. Successful outcome in patients with acute lung injury/ARDS. Chest 1999; 115: 173-177.

40. Antonelli M, Conti G, Rocco M. A comparison of noninvasive positive-pressure ventilation and conventional mechanical ventilation in patients with acute respiratory failure. N Engl J Med 1998; 339: 429-435.

41. Appendini L, Pasessio A, Zanaboni S. Physiologic effects of positive end-expiratory pressure and mask pressure support during exacerbations of chronic obstructive pulmonary disease. Am J Respir Crit Care Med 1994; 149: 1069-1076.

42. Comstock GW. Further comments on problems in death certification. Am J Epidemiol 1986; 124(2): 180-181. 